\title{
Discussion to paper of Gibbon et al.
}

\author{
Chairman: DR H. J. HACHEN
}

Chairman. Mr Gibbon's paper is very important because although much is known about neurophysiology the influence of the catecholamines upon the proximal urethra is very little understood. His findings partly explain why even in patients with upper motor neurone lesions we have sometimes had good results with these tests and that the presence of alpha receptors at the level of the urethral sphincter is more important than hitherto suspected. I would like to ask him, when he indicated the values in the urethral pressure profile whether this was maximal urethral pressure, because I would have liked to see the shape of the curve of urethral profile not only the value. How are you sure that your catheter tip is really at the level of the striated sphincter when you are measuring the maximal pressure?

MR GIBBON (G.B.). In a short paper each individual change in the urethral pressure profile cannot be shown; the mean of 6 or so was given at the different levels. We did not check the position of the eye of the catheter radiologically. These were purely functional studies and we made a point of showing that we are talking about altered function, not trying to distinguish between the striated muscle and the plain muscle because we do not know how much of the striated muscle activity is under sympathetic control rather than somatic control. Certainly in the lower motor neurone lesions in which the abnormal urethral response is very strong indeed one would expect the somatic element to be absent, but the shape of the profile suggests that it is the striated muscle which may be dominant in this change.

ChaIRMan. Have you had any opportunities to study the effects of the catecholamine release during dysreflexia in patients on the urethral sphincter profile? Sometimes patients in the state of hyperreflexic situations encounter major difficulties in initiating voiding and if dysreflexia is due to bladder retention this sometimes obliges us to make a catheterisation because the patient in a dysreflectic stage is unable to initiate micturition, and this may be in relation to excessive sphincter activity due to catecholamine release.

MR GIBBON. I think there is a lot of further work to be done and we haven't in fact attempted to measure catecholamines during hyperreflexia but I think other people have shown that the hyperreflexia is in fact due to the catecholamine release and often the bladder is the source of the problem.

Mr I. NusEIBEH (G.B.). I share with Mr Gibbon the understanding of the somatic and the autonomic influence on the urethra, but from our experience we know that with patients with high residual urine we do external sphincterotomy. We may combine it with transurethral resection of the bladder neck but in spite of that there is always residual urine in a certain number of patients. Can I add that there is another factor to the outflow of suction in the bladder itself in the neuropathic bladder that is a mechanical factor due to changes in the position of the bladder and probably its rotation. From our experience when patients with neuropathic bladder come to us, we found their bladder most of the time was lying in the right iliac fossa. It is not towards the umbilicus as it is in the prostatic hypertrophy. Am I right in suggesting that, Mr Gibbon?

MR GIBBON. I would agree entirely that the voiding is a demonstration of the balance between the outflow pressure and the urethral resistance and only if one does full urodynamic studies does one find out what part the detrusor is actually playing. Not uncommonly the detrusor starts off very strongly with a good contraction and simple cystometrography would suggest that the detrusor function is normal. This detrusor activity fades quite rapidly and leaves the patient with a poor emptying, although the urethral pressure profiles may appear to be at a low value. Unless you do the urethral pressure profiles in an upright position you are not really getting a true picture of what 
is happening when the patient is trying to void. Sometimes in the elderly prostatic patient the bladder also goes to the right rather than up in the midline. I don't know what the significance of that is functionally.

ChAIRMAN. Regarding the importance of alpha-adrenergic receptors at the level of the striated sphincter there just came out an interesting paper by Naninga and Kaplan and they were treating patients with dysreflexia with injections of regertine, glutonamine CIBA. They noticed, I quote, 'Shortly after the intravenous injection of regertine there was a decrease of perineal muscle EMG activity. It was noted also that the bladder had not yet been drained and the bladder pressure remained elevated. Therefore during subsequent cystometry and perineal EMG evaluation in three other patients particular attention was focussed on the EMG activity when pentolamine was administered for autonomic hyperreflexia'. The paper ends with a summary saying 'the effect of alpha adrenergic blocking agent pantolamine was studied in three patients with dysreflexia the drug reduced the EMG activity in all these patients'. This clearly suggests that the normal suppression of the external sphincter activity during micturition might be related to a reduction in the external sphincter activity supplied by the sympathetic system, so it shows indirectly the importance of sympathetic innervation of the striated sphincter.

Dr PERKASH (U.S.A.). I am tempted to answer the second part because I have been interested in the alpha-adrenergic activity in relation with spinal injury patients and I do routine intravenous pentolamine testing on all these patients. In the past four years I've done about 4000 studies. There is a paper that I published in the fournal of Urology in the month of June '79 on the effect of the reduction in EMG activity of the striated sphincter after Pentolamine. It does not really mean that sympathetic innervation is there for the striated muscle, what it indicates is that alpha blocking with intravenous Pentolamine, leads to relaxation of the bladder neck. Urine that enters into the first part of the urethra sets up the reflex which relax the periurethral striated sphincter. I do not agree with the findings and conclusions that Dr Hachen has quoted from Dr Naninga's paper that it indicates that sympathetic fibres supply the striated sphincter. There is no real evidence so far to prove that. On the other hand it indicates that relaxing the bladder neck has set up a micturition reflex which relaxes the urethral striated sphincter. We do not have direct evidence to prove that. Getting back to Mr Gibbon's paper, what I wanted to know from Mr Gibbon is, was there a demonstrable rise in catecholamines and what is the mechanism if you put a cuff on the left thigh? How could you explain that the catecholamines will increase? I thought you mentioned that the catecholamines were higher in those patients.

Mr GibBon. When we put on the thigh cuffs we waited for 3 to 4 minutes and then re-estimated the serum catecholamines and the usual rise was found which one would expect from the tendency for the blood pressure to fall.

ChaIrman. Did you use pressure around the diastolic value or about the systolic because about the systolic you get the hyperactive hyperaemia and catecholamine release, whereas if you are below or just at diastolic pressure you increase the renal effective pressure in the distal segment because you block the return but usually there is no catecholamine release in these patients.

Mr Gibbon. This was purely to block the venous resturn and the fall in blood pressure which would be expected of course was compensated except in the cervical lesions, but the change in the urethral pressure profile occurred whilst the thigh cuffs were still inflated, not after they had been released. We assume that we are in fact simulating cardiovascular changes which normally occur with a change in posture to the upright position. I think there is a good deal of evidence from Koyigani in Japan, who injecting noradrenaline into paraplegic patients showed exactly the same increase in urethral pressure profile as we have shown by putting thigh cuffs on. I agree there is a lot more work to be done yet before we can say this is proved.

DR MAURY (France). Is there a normal physiological explanation for the bladder neck always being open during detrusor filling in some patients with sacral lesions.

MR GIBBON. This was in low motor neurone lesions?

Dr MAURY. Yes. 
Mr Gibbon. This was first pointed out by Watkins in the Hunterian Lecture on this very subject in Manchester in 1934, namely that in the lower motor neurone region the obstruction was commonly at the level of the striated sphincter or membranous urethra not at the bladder neck. The bladder neck opens when the bladder fills up and the pressure becomes sufficient or if the patient strains or uses the Crèdè manoeuvre, the obstruction is lower down. Now what he could not demonstrate was what the obstruction was due to, but recent work shows that the obstruction is spasticity of the urethral muscle and it is in fact related to posture, and if you measure the urethral resistance with the patient lying flat you will see that it is very low, but patients don't void lying down. You've got to do the resistance with the patient sitting up.

ChaIRMan. We know the importance of posture in evaluating incontinence in the female, for instance, but in the spinal injured patients this has been very largely neglected. Most papers have been published on patients prone on the urological table. Both your observations and the ones by Dr Madersbacher are very important. We know that flow metric evaluation is difficult in spinal injured patients because they have the difficulty of initiating micturition, but I think that your result and Dr Madersbacher's seemed to show the enormous importance of change of position and the elasticity of various segments in the urethra.

No NAME GIVEN. I fully agree that there are sympathetic reflex mechanisms. But I think we cannot overlook the mechanical component. Even if you have the patient voiding by straining-Crèdè-in the lying position you have the typical deformation of the urethra where it passes the pelvic floor, and one cannot forget that the urethra at that point is surrounded by a bulky mass of pelvic floor muscle and there is I am sure to some extent also a mechanical effect additionally to the functional, as you have mentioned.

Mr GibBon. Yes I think that Dr Hachen has confirmed this in showing the different response in the different age groups. One would expect the pelvic floor to be perhaps more resistant in the older age groups.

SIR LudWIG GutTManN (G.B.). In our bladder distension experiments since 1947 we came across a dissociation between adrenalin and noradrenalin which sometimes was very marked. There is no doubt that the adrenalin starts first to go up and then the noradrenalin follows, which has much higher values. How far have you come across this in your experiments? That is the first question I want to ask. Secondly, of course, this change in the urethral function on positioning is very well known but your experiments have really confirmed how important the position is for the proper function of the muscular system of the bladder as I have established for the muscular system of the lower limbs during spinal shock and after return of the reflex activity of the spinal cord later.

How far have you got experience already in people with increased age and long standing paraplegia, in particular concerning the perspective size and function and the benefits of prostatectomy.

Mr GIBBON. Sir Ludwig is very knowledgeable about catecholamine changes in paraplegia. The main changes are noradrenalin rather than adrenalin. We have not attempted to distinguish the values in the different age groups; as I said we are considering the mean values. I think that certainly if an elderly man has got a prostatic obstruction which can be demonstrated urodynamically we would resect the whole of the adenomatous prostate rather than just do a bladder neck resection for him.

DR MADERSBACHER (Austria). May I add one comment to your point. If you have a paraplegic patient with a prostatic hypertrophy and a sphincter detrusor dysfunction, you should do a TUR the prostate together with sphincterotomy. Otherwise-we had one or two of these cases-the functional obstruction of the pelvic floor exists. I would always stress that if there is a significant detrusor sphincter dyssinergia in these patients I would always combine both procedures.

Mr GibBon. I would agree entirely with that.

DR PERKash (U.S.A.). If Pentolamine shows marked alpha-adrenergic at the bladder neck and there is also detrusor periurethral striated sphincter dyssynergia, I prefer to divide the bladder neck and the sphincterotomy as the results concerning the effects on autonomic dysreflexia were much better and further surgery was not needed. 